\title{
Perfil de personalidad y consumo de drogas en adolescentes escolarizados
}

\author{
Marina Beatriz Fantin* \\ Facultad de Ciencias Humanas, Universidad Nacional de San Luis, Argentina \\ * Licenciada en Psicología. \\ Enviar correspondencia a: \\ Marina B. Fantin, Pringles 1394, San Luis. Argentina. Correo electrónico: mbfantin@unsl.edu.ar
}

Recibido: 4 de octubre de 2005. Aceptado: 7 de abril de 2006

\section{RESUMEN}

El objetivo de la presente investigación es describir e identificar estilos de personalidad, preocupaciones y síndromes clínicos en una muestra de adolescentes escolarizados de la ciudad de San Luis, Argentina, realizando una aproximación a la problemática de las adicciones. Se indaga la proporción de adolescentes que presentan patrones de consumo de sustancias adictivas, con la finalidad de establecer comparaciones entre grupos de consumidores y de no consumidores. Se utilizaron tres instrumentos de evaluación: el Inventario Clínico para Adolescentes, de Theodore Millon (MACl, 1993), el Test de Identificación de Desórdenes en el Uso del Alcohol (AUDIT, OMS, 1993) y el Cuestionario de Identificación de Dependencia de las Drogas (CIDD), elaborado ad-hoc. La aplicación de esta batería a una muestra de 203 adolescentes escolarizados, hombres y mujeres entre 15 y 19 años de edad, se efectuó en los meses de junio a septiembre de 2004. Los resultados indican que entre las sustancias adictivas más consumidas por los adolescentes de la muestra se encuentran el alcohol y el tabaco. Aproximadamente dos de cada tres adolescentes ha consumido alcohol a lo largo de su vida, y cerca de un $18 \%$ de la población total presenta un consumo perjudicial o de riesgo de esta sustancia. Los resultados señalan que el grupo consumidor de sustancias obtiene diferencias significativas, respecto a los no consumidores, en casi todas las escalas utilizadas para evaluar personalidad, observándose un patrón distinto en unos y otros.

\section{ABSTRACT}

The objective of this research is to describe and identify personality styles, concerns, and clinical syndromes in adolescents in San Luis City, Argentina, and to make an approach to the problems of addiction. The proportion of adolescents presenting addictive substance use patterns is calculated in order to establish comparisons between user and nonuser groups. Three assessment instruments were used: The Millon Adolescent Clinical Inventory (MACl, 1993), the Alcohol Use Disorders Identification Test (AUDIT, OMS; 1993) and an ad-hoc Drug Addiction Identification Questionnaire (DAIQ). These were administered to a sample of 203 school adolescents, males and females between 15 and 19 years of age, in the period between June and September 2004. Results show that alcohol and tobacco are the addictive substances most frequently used by the adolescents in the sample. Approximately two out of three adolescents have consumed alcohol in their lifetime, and $18 \%$ of the population consumes a harmful or risky amount of this substance. The results suggest that significant differences exist between the group of adolescent substance users and the nonuser group on almost every scale used to evaluate personality, a different pattern being observed in each group.

Key words: Personality - Adolescents - Addiction.

Palabras clave: Personalidad adolescentes, adicciones.

\section{INTRODUCCIÓN}

n relación a las investigaciones que buscan establecer un vínculo entre estilos de personalidad y el uso de drogas, las teorías psicológicas generalmente enfatizan aspectos psicológicos individuales como baja autoestima, mayor ansiedad y menor capacidad de control en adolescentes consumidores. Aún así, hasta la fecha no se ha podido demostrar la existencia de una personalidad adictiva o perfil específico relacionado con las conductas de adicción, aunque sí ciertas características particulares (Mesa y León-Fuentes, 1996). La observación clínica constata que existen ciertas características individuales y fac- 
tores de vulnerabilidad personal que pueden favorecer el consumo o predisponer a dicha conducta: "...la inmadurez, la dependencia de su familia e iguales, no asunción de responsabilidades, inseguridad, baja confianza en sus capacidades, alto grado de paranoidismo, falta de motivación e iniciativa, conducta extraña, aislamiento de la familia, escasa o nula comunicación, inestabilidad e irritabilidad emocional, baja resistencia a la frustración" (Mesa y Leon-Fuentes, 1996). Mezzich, Tarter, Giancola, Lu, Kirisci y Parks (1997) señalan otros aspectos como la desregulación comportamental, la afectividad negativa y la victimización infantil relacionados al uso de sustancias.

La adolescencia es considerada una etapa delimitada entre la pubertad y la madurez, en donde suceden cambios y transiciones que concluyen en un individuo adulto, preparado para enfrentar un trabajo, una familia y nuevas responsabilidades. "Un adolescente es un ser humano que pasó la pubertad y que todavía se encuentra en etapa de formación ya sea en lo referente a su capacitación profesional, a la estructuración de su personalidad o a la identidad sexual" (Obiols y Obiols, 1994).

Desde una perspectiva evolutiva, la adolescencia comprende diferentes períodos, cada uno con características específicas que los distinguen. Teniendo en cuenta las teorías psicológicas sobre la adolescencia, diversos autores identifican el período de la adolescencia inicial o temprana (se ubicaría en un rango muy amplio de edad debido a que el comienzo es muy variable en cada persona, pero aproximadamente entre los 11 y los 14 años), la etapa de la adolescencia media (14-15 a 17 años) y por último la adolescencia final (17 años en adelante, no hay un límite superior de edad consensuado entre autores). Generalmente hacia la adolescencia final se considera que el adolescente está en camino de completar las tareas esperadas por los padres y la sociedad: el logro de una identidad madura, una identidad sexual adecuada, un comportamiento socialmente responsable, la independencia de los padres y la capacidad de establecer relaciones nuevas y más maduras con los demás (Florenzano, 1992; Peláez y Luengo, 1996).

Desde el enfoque individual o psicológico, el análisis del consumo de sustancias en la adolescencia se centra en las motivaciones que dan lugar al acercamiento a las drogas (curiosidad, intentos de evasión, desinhibición, búsqueda de afectos positivos, presión del grupo, desafío, ansiedad, etc.) y en los sentimientos de los adolescentes: la angustia y el malestar; la experiencia de duelo o pérdida afectiva que lleva a buscar estímulos externos placenteros; la búsqueda de identidad y de diferenciación radical de los otros (Mesa y León-Fuentes, 1996).

La mayoría de los autores que investigan la problemática coinciden en afirmar que la adolescencia "...se ha ido constituyendo progresivamente como una edad de alto riesgo para el abuso de sustancias adictivas, y cada vez en edades más tempranas" (Mesa y LeónFuentes, 1996). Esta afirmación se encuentra respaldada por varias razones: pobre autoestima, escaso desarrollo de las capacidades, predominio de locus de control externo, carencia de autodisciplina, dificultad para las relaciones interpersonales, pobre capacidad de juicio, escaso sentido de responsabilidad, personalidad lábil. Si a ello se une una familia que no supo poner límites, o con graves problemas de autoridad (por exceso o defecto) el riesgo se encuentra potencialmente aumentado (Glenn, 1987).

En 1969, Millon delinea su modelo de la personalidad introduciendo un marco conceptual para describir el desarrollo y mantenimiento de la personalidad basado en tres ejes: activo-pasivo, placer-dolor y yo-otros. En su teoría, el autor entrecruza el eje activo-pasivo con cuatro estrategias de reforzamiento (desapegado, dependiente, independiente y ambivalente) y obtiene ocho patrones básicos de personalidad (introvertido, inhibido, sumiso, sociable, narcisista, agresivo, conformista y negativista). Posteriormente, las descripciones encontradas en 1969 fueron ampliadas y enriquecidas (Strack, 1999).

En el modelo biosocial, Millon considera que los determinantes principales del estilo de personalidad que los individuos desarrollan son dos: la constitución biofísica y las experiencias pasadas. El estilo de personalidad que un niño desarrolla en contacto con el mundo está enraizado inicialmente en los factores básicos constitucionales. Esto constituye un conjunto de capacidades y disposiciones que colorean la forma en que el niño percibe los eventos y determina el carácter de las respuestas a los mismos: "Bajo condiciones ambientales normales un niño encontrará formas de relacionar los eventos que sean adecuadas a su disposición constitucional. Sin embargo, si la presión y demandas son demasiado severas, éstas pueden forzar al niño a desarrollar estrategias potencialmente problemáticas o contrarias a sus inclinaciones naturales" (Davis, 1999).

Teniendo como base la teoría de personalidad de Millon se utilizará en el presente estudio la aplicación del instrumento del mismo autor para evaluar estilos de personalidad en los adolescentes y síndromes clínicos relacionados al abuso de sustancias.

Los objetivos del presente estudio son:

- Realizar una aproximación a la problemática de las adicciones en la población adolescente de la Ciudad de San Luis.

- Indagar porcentajes de adolescentes que presentan patrones de consumo de sustancias adictivas.

- Conocer estilos de personalidad, preocupaciones y síndromes clínicos de la población en estudio. 
- Comparar grupos de adolescentes consumidores con aquellos no consumidores.

\section{MÉTODO}

\section{Sujetos}

La muestra estuvo conformada por 203 adolescentes de ambos sexos, de edades comprendidas entre 15 y 19 años (media $=16,58$ y desviación estándar $=$ $1,02)$ del nivel polimodal de escuelas públicas de la ciudad de San Luis. En cuanto al género, la muestra se compone de 74 varones y 129 mujeres.

Los sujetos fueron extraídos de población normal de adolescentes de la Ciudad de San Luis. Para tal fin se estableció en primer lugar trabajar con las escuelas de la ciudad que cumplieran con el requisito del nivel polimodal. Se obtuvo una lista con todas las escuelas públicas y privadas. En este caso, se decidió realizar una categorización de escuelas en relación a un criterio específico que fue determinado como el Nivel Socio-económico de cada una, buscando la forma de seleccionar a las escuelas de manera que todas pudieran tener la misma posibilidad de inclusión en la muestra. Se agrupó la totalidad de escuelas por criterios (muestreo estratificado), por zonas barriales de la ciudad y también por nivel socio-económico, extrayéndose 5 grandes categorías: Escuelas de Niveles Bajos, Escuelas de Niveles Medios-Bajos, Escuelas de Niveles Medios, Escuelas de Niveles Sociales Mixtos y Escuelas Privadas (de Niveles Medios-Altos). Finalmente, fueron sorteadas dos escuelas en cada categoría, utilizando el muestreo simple o azaroso para la selección de las mismas. De esta manera, se incluyó un total de 10 escuelas conformando la muestra actual.

\section{Instrumentos}

\section{Inventario Clínico para Adolescentes de Millon (MACI, 1993).}

Se compone de 27 escalas, que identifican estilos de personalidad básicos, preocupaciones personales y actitudes, y síntomas clínicos de los adolescentes emocionalmente perturbados. Las escalas de personalidad son las siguientes: Introvertido, Inhibido, Depresivo, Sumiso, Dramático, Narcisista, Antisocial, Sádico, Conformista, Oposicionista, Autoderrotista, Borderline. Las escalas de preocupación manifiesta son: Difusión de la Identidad, Autodesvalorización, Malestar Corporal, Disconfort Sexual, Inseguridad Pares, Insensibilidad Social, Discordia Familiar, Abuso Infantil. Las escalas de síndromes clínicos son: Desórdenes Alimenticios, Abuso de Sustancias, Delincuencia, Impulsividad, Ansiedad, Depresión, Tendencia Suicida. Existe, además, una escala que se relacionan con la validez y confiabilidad del test: Confiabilidad. La fiabilidad de las escalas oscila entre un Coeficiente Alfa de Cronbach de 0,73 a 0,91. El Alfa de Cronbach total de la prueba es de 0,82.

\section{Test de Identificación de Desórdenes en el Uso de Alcohol (AUDIT), elaborado por la Organiza- ción Mundial de la Salud, (1993).}

Consta de diez preguntas que evalúan las conductas en relación con el alcohol a través de una escala de tipo Lickert. El rango de las puntuaciones es de 0 a 40; puntuaciones mayores de 20 orientan hacia la existencia de dependencia plena respecto al alcohol; entre 8 y 20 puntos, señalan un consumo perjudicial o de riesgo y una puntuación inferior a 8 no refleja dificultades en el consumo de la sustancia. Los autores definen los criterios anteriormente citados de dependencia y consumo perjudicial en relación a cuatro aspectos: la cuantificación del consumo alcohólico (cantidad en Unidades Básicas Estándar de alcohol y frecuencia de consumo), el comportamiento o actitud ante la bebida, las reacciones adversas o consecuencias de su consumo para la persona y la toma de conciencia del problema.

En cuanto a las propiedades psicométricas, la confiabilidad del instrumento ha sido estimada mediante el Coeficiente Alfa de Cronbach $(0,80)$ y la validez mediante el índice de correlación con el Test de Screening de Alcoholismo de Michigan (MAST; Selzer, 1971), ( $r=0,88)$ y con determinaciones analíticas como la Gamaglutamiltranspeptidasa (GGT; Riggs, 1998), ( $r=0,31$ en varones y 0,46 en mujeres) que es un marcador biológico sobre el nivel de alcohol existente.

\section{Cuestionario de Identificación de Dependencia de las Drogas (CIDD), elaborado ad-hoc.}

Consta de 10 preguntas que indagan la relación de los adolescentes con las conductas de uso, abuso o dependencia de sustancias adictivas en general. El formato de respuesta es combinado, algunos ítems tienen respuesta sí-no, otros múltiple opción y por último algunos con escala de tipo Lickert. La evaluación del cuestionario es cualitativa. En cuanto a las propiedades psicométricas, el instrumento presenta una confiabilidad de 0,80 estimada según el Coeficiente Alfa de Cronbach. La validez del mismo aún no ha sido obtenida.

\section{Procedimiento}

Para el análisis de los datos se obtuvieron frecuencias y porcentajes de las variables en estudio, y media, desviación estándar y diferencia de medias a través del paquete estadístico SPSS. 


\section{RESULTADOS}

Tabla 1. Frecuencia y porcentaje obtenido en la variable edad

\begin{tabular}{|c|c|c|}
\hline Edad & Frecuencia & Porcentaje \\
\hline 15 años & 36 & 17,7 \\
\hline 16 años & 50 & 24,6 \\
\hline $\mathbf{1 7}$ años & 87 & 42,9 \\
\hline $\mathbf{1 8}$ años & 23 & 11,3 \\
\hline 19 años & 7 & 3,4 \\
\hline Total & 203 & 100,0 \\
\hline
\end{tabular}

En la tabla 1 se observa que un $42,9 \%$ de la muestra son adolescentes de 17 años, le siguen en porcentajes los adolescentes de 16 años (24,6\%); los de 15 años $(17,7 \%)$ y por último el grupo de adolescentes de 18 años con un $11,3 \%$. Un porcentaje muy bajo $(3,4 \%)$ de adolescentes de 19 años se encuentra representado en la muestra.

Variables del Test de Identificación de Desórdenes en el Uso del Alcohol (AUDIT)

Tabla 2. Frecuencia y porcentaje obtenidos mediante la aplicación del Test de Identificación de Desórdenes en el Uso de Alcohol (AUDIT)

\begin{tabular}{|l|c|c|}
\hline & Frecuencia & Porcentaje \\
\hline $\begin{array}{l}\text { Consumo no } \\
\text { perjudicial }\end{array}$ & 166 & 81,8 \\
\hline $\begin{array}{l}\text { Consumo } \\
\text { perjudicial }\end{array}$ & 35 & 17,2 \\
\hline $\begin{array}{l}\text { Dependencia } \\
\text { plena }\end{array}$ & 2 & 1,0 \\
\hline Total & 203 & 100,0 \\
\hline
\end{tabular}

Los datos observados en la tabla 2, que refieren a los resultados obtenidos del Test de Identificación de Desórdenes en el Uso del Alcohol (AUDIT), señalan que el $81,8 \%$ de los adolescentes de la muestra no presenta dificultades en el consumo de alcohol, mientras que el $17,2 \%$ presenta un consumo perjudicial o de riesgo, y por último un 1\% de los adolescentes de la muestra presentaría una dependencia plena respecto al alcohol, sin control sobre el mismo cuando se ingiere.
Tabla 3. Frecuencia y porcentaje obtenidos en la variable frecuencia en el consumo de alcohol

\begin{tabular}{|l|c|c|}
\hline & Frecuencia & Porcentaje \\
\hline Nunca & 74 & 36,4 \\
\hline $\begin{array}{l}\text { Mensualmente o } \\
\text { menos }\end{array}$ & 73 & 36,0 \\
\hline $\begin{array}{l}\text { Entre 2 y 4 veces } \\
\text { al mes }\end{array}$ & 42 & 20,7 \\
\hline $\begin{array}{l}\text { Entre 2 y 3 veces } \\
\text { a la semana }\end{array}$ & 10 & 4,9 \\
\hline $\begin{array}{l}\text { 4 o más veces } \\
\text { a la semana }\end{array}$ & 4 & 2,0 \\
\hline Total & 203 & 100,0 \\
\hline
\end{tabular}

En la Tabla 3 se observa que el $36,4 \%$ de los sujetos entrevistados manifiestan no consumir alcohol, lo que indica que existe un $63,6 \%$ que sí lo hace, de los cuales el 36,0\% mantiene un consumo mínimo (menos de una vez por mes), el $20,7 \%$ consume entre dos y cuatro veces por mes y el 6,9\% de los adolescentes bebe desde dos hasta cuatro o más veces por semana.

Variables del Cuestionario de Identificación de Dependencia a las Drogas (CIDD)

Tabla 4. Frecuencia y porcentaje obtenido en la variable tipos de sustancias que se consumen actualmente del Cuestionario de Identificación de Dependencia a las Drogas (CIDD)

\begin{tabular}{|l|c|c|}
\hline & Frecuencia & Porcentaje \\
\hline Alcohol & 66 & 32,5 \\
\hline Tabaco & 62 & 30,5 \\
\hline LSD & 2 & 1,0 \\
\hline Marihuana & 16 & 7,9 \\
\hline Cocaína & 3 & 1,5 \\
\hline $\begin{array}{l}\text { Opio, Heroína, } \\
\text { Mezcalina }\end{array}$ & 0 & 0 \\
\hline $\begin{array}{l}\text { Inhalantes, } \\
\text { solventes, } \\
\text { pegamentos }\end{array}$ & 1 & 0,5 \\
\hline $\begin{array}{l}\text { Medicamentos, } \\
\text { sedantes, } \\
\text { ansiolíticos, } \\
\text { tranquilizantes }\end{array}$ & 7 & 3,4 \\
\hline Otros & 2 & 1,0 \\
\hline
\end{tabular}


Al ser interrogados sobre el consumo de sustancias que mantienen en la actualidad, los adolescentes (tabla 4) respondieron que el 32,5\% consume alcohol y el 30,5\% tabaco. En menor medida, el 7,9\% consume marihuana y el 3,4\% consume medicamentos (ansiolíticos, sedantes, tranquilizantes, etc.), el 1,5\% cocaína, un 0,5\% inhalantes, solventes y pegamentos y un $1 \%$ otras drogas.

Tabla 5. Porcentaje obtenido en la variable frecuencia de consumo actual de drogas del Cuestionario de Identificación de Dependencia a las Drogas (CIDD)

\begin{tabular}{|l|c|}
\hline & Porcentaje \\
\hline No llega a una vez por mes & 17,74 \\
\hline Mensualmente & 22,58 \\
\hline Semanalmente & 22,58 \\
\hline Diariamente & 37,10 \\
\hline
\end{tabular}

De la totalidad de adolescentes que consumen en la actualidad drogas un $17,74 \%$ consume menos de una vez por mes, el 22,58\% lo hace mensualmente, el mismo porcentaje de sujetos consume semanalmente y el $37,10 \%$ consume sustancias diariamente (tabla 5)

Tabla 6. Frecuencia y porcentaje obtenido en la variable razones para el consumo del Cuestionario de Identificación de Dependencia a las Drogas (CIDD)

\begin{tabular}{|l|c|}
\hline & Porcentaje \\
\hline Para experimentar & 21,2 \\
\hline Para divertirse & 18,2 \\
\hline Para no sentirse rechazado & 0 \\
\hline No puede abandonarla & 3,0 \\
\hline Otro & 2,0 \\
\hline
\end{tabular}

La tabla 6 señala que los adolescentes de la muestra justifican el consumo de sustancias alegando principalmente dos razones: probar, experimentar (43\%) y sentirse cómodo o divertirse en situaciones sociales $(18,2 \%)$. El 3\% de los sujetos responden que la razón para el consumo es que no pueden abandonarla y la necesitan diariamente. Variables del Inventario Clínico de Personalidad Adolescente de Millon (MACI)

Tabla 7. Media, desviación estándar y diferencia de medias obtenidas en la variable puntaje total del AUDIT y variables del Inventario Clínico de Personalidad Adolescente de Millon, en la población total y entre grupos de consumidores y no consumidores de sustancias

\begin{tabular}{|l|c|c|c|c|c|c|c|c|}
\hline \multirow{2}{*}{$\begin{array}{l}\text { Muestra } \\
\text { Variable }\end{array}$} & \multicolumn{2}{|c|}{$\begin{array}{c}\text { Muestra total } \\
\text { (N=203) }\end{array}$} & \multicolumn{2}{c|}{ Probó (N =92) } & \multicolumn{2}{c|}{$\begin{array}{c}\text { No probó } \\
\text { (N = 111) }\end{array}$} & \multicolumn{2}{c|}{ Prueba t } \\
\cline { 2 - 9 } & Media & $\mathbf{D . S}$ & Media & $\mathbf{D . ~ S}$ & Media & D. S & t & p \\
\hline Puntaje total AUDIT & 3,43 & 4,56 & 6,01 & 5,12 & 1,29 & 2,51 & 8,56 & $\mathbf{0 , 0 0 0}$ \\
\hline MACI: Sumiso & 67,18 & 14,32 & 62,32 & 15,04 & 71,22 & 12,38 & $-4,62$ & $\mathbf{0 , 0 0 0}$ \\
\hline Antisocial & 55,92 & 18,10 & 61,41 & 18,88 & 51,37 & 16,15 & 4,08 & $\mathbf{0 , 0 0 0}$ \\
\hline Sádico & 33,54 & 23,65 & 42,41 & 26,94 & 26,19 & 17,50 & 5,16 & $\mathbf{0 , 0 0 0}$ \\
\hline Conformista & 52,16 & 12,23 & 48,45 & 13,60 & 55,23 & 10,02 & $-4,08$ & $\mathbf{0 , 0 0 0}$ \\
\hline Oposicionista & 54,12 & 20,25 & 58,87 & 21,26 & 50,19 & 18,56 & 3,10 & $\mathbf{0 , 0 0 2}$ \\
\hline Autoderrotista & 47,84 & 25,59 & 52,58 & 27,83 & 43,92 & 22,97 & 2,42 & $\mathbf{0 , 0 1 6}$ \\
\hline Borderline & 49,41 & 23,96 & 53,66 & 24,83 & 45,89 & 22,73 & 2,32 & $\mathbf{0 , 0 2 1}$ \\
\hline Disconfort Sexual & 60,40 & 14,31 & 56,48 & 15,12 & 63,65 & 12,79 & $-3,66$ & $\mathbf{0 , 0 0 0}$ \\
\hline Insensibilidad Social & 46,80 & 16,84 & 51,87 & 18,12 & 42,60 & 14,48 & 4,04 & $\mathbf{0 , 0 0 0}$ \\
\hline Discordia Familiar & 65,39 & 20,51 & 71,70 & 19,32 & 60,17 & 20,07 & 4,14 & $\mathbf{0 , 0 0 0}$ \\
\hline Abuso Infantil & 40,19 & 21,59 & 45,04 & 23,11 & 36,16 & 19,45 & 2,97 & $\mathbf{0 , 0 0 3}$ \\
\hline Abuso de Sustancias & 27,98 & 19,58 & 36,64 & 23,43 & 20,80 & 11,61 & 6,25 & $\mathbf{0 , 0 0 0}$ \\
\hline Delincuencia & 45,87 & 15,66 & 50,32 & 18,06 & 42,19 & 12,27 & 3,80 & $\mathbf{0 , 0 0 0}$ \\
\hline Impulsividad & 49,60 & 21,08 & 57,32 & 20,61 & 43,21 & 19,33 & 5,02 & $\mathbf{0 , 0 0 0}$ \\
\hline Ansiedad & 74,42 & 19,74 & 67,52 & 18,89 & 80,14 & 18,64 & $-4,77$ & $\mathbf{0 , 0 0 0}$ \\
\hline
\end{tabular}


Los resultados de la Tabla 7 permiten hacer una descripción global de los adolescentes que componen la muestra y, además, una discriminación según hayan probado sustancias alguna vez en su vida o no. En casi todas las escalas hay diferencias significativas entre consumidores y no consumidores. Las diferencias de media altamente significativas entre ambos grupos señalan que el grupo consumidor de sustancias tiende a presentar, en mayor medida que los no consumidores, rasgos de personalidad Antisocial, Oposicionista,
Sádico, Autoderrotista y Borderline; mayores conflictos relacionados con Insensibilidad Social, Discordia Familiar, Abuso Infantil, Abuso de Sustancias, Delincuencia e Impulsividad, y puntuaciones más elevadas en el Test de Identificación de desórdenes en el uso del Alcohol (AUDIT); en tanto que el grupo de personas no consumidoras de sustancias presentan mayores puntuaciones en las escalas Sumiso, Conformista, Disconfort Sexual y Ansiedad.

Tabla 8. Diferencia de media obtenida en la variable puntaje total del AUDIT y variables del Inventario Clínico de Personalidad Adolescente de Millon en mujeres y varones

\begin{tabular}{|l|c|c|c|c|c|c|}
\hline \multirow{2}{*}{$\begin{array}{l}\text { Muestra } \\
\text { Variables }\end{array}$} & \multicolumn{2}{|c|}{$\begin{array}{c}\text { Masculino } \\
\text { (N=74) }\end{array}$} & \multicolumn{2}{c|}{$\begin{array}{c}\text { Femenino } \\
\text { (N= 129) }\end{array}$} & \multicolumn{2}{c|}{ Prueba t } \\
\cline { 2 - 7 } & Media & D. S & Media & D. S & t & p \\
\hline Puntaje total AUDIT & 6,50 & 6,20 & 3,46 & 4,57 & 8,56 & $\mathbf{0 , 0 0 0}$ \\
\hline MACI: Introvertido & 54,31 & 15,44 & 59,94 & 13,89 & $-5,74$ & $\mathbf{0 , 0 0 0}$ \\
\hline Inhibido & 49,95 & 22,33 & 62,85 & 22,93 & $-8,39$ & $\mathbf{0 , 0 0 0}$ \\
\hline Depresivo & 45,83 & 25,56 & 57,53 & 27,93 & $-6,38$ & $\mathbf{0 , 0 0 0}$ \\
\hline Sumiso & 59,64 & 11,89 & 67,75 & 14,67 & $-8,74$ & $\mathbf{0 , 0 0 0}$ \\
\hline Dramático & 56,37 & 14,45 & 50,64 & 14,41 & 5,87 & $\mathbf{0 , 0 0 0}$ \\
\hline Narcisista & 49,90 & 14,87 & 41,43 & 15,03 & 8,36 & $\mathbf{0 , 0 0 0}$ \\
\hline Antisocial & 63,42 & 16,71 & 55,50 & 17,57 & 6,78 & $\mathbf{0 , 0 0 0}$ \\
\hline Sádico & 43,26 & 23,57 & 32,90 & 22,95 & 6,60 & $\mathbf{0 , 0 0 0}$ \\
\hline Autoderrotista & 44,89 & 24,32 & 53,83 & 26,18 & $-5,17$ & $\mathbf{0 , 0 0 0}$ \\
\hline Borderline & 45,73 & 20,29 & 54,20 & 34,51 & $-4,17$ & $\mathbf{0 , 0 0 0}$ \\
\hline
\end{tabular}

Se encontraron diferencias de media significativas en cuanto a la variable sexo en casi todas las escalas utilizadas. En las puntuaciones totales del Test de Identificación de Desórdenes en el Uso de Alcohol (tabla 8), en donde se observa que los varones tienden a presentar puntuaciones más elevadas que las adolescentes mujeres; es decir que existiría una tendencia más marcada a consumir alcohol en el sexo masculino.

Se observa, en relación a los estilos de personalidad, que las mujeres se caracterizan por ser más Introvertidas, Inhibidas, Depresivas, Sumisas, Autoderrotistas y Borderline, en relación a los varones, mientras que éstos tienden a presentar mayores puntuaciones que las mujeres en los estilos de personalidad Dramático, Narcisista, Antisocial y Sádico.

\section{DISCUSIÓN}

Uno de los objetivos de este trabajo hace referencia a la aproximación al conocimiento de la población adolescente de la ciudad de San Luis. Para ello, resulta fundamental describir las características generales de nuestra población. San Luis es una pequeña ciudad del interior del país, con aproximadamente 140.000 habitantes, que ha experimentado un crecimiento poblacional significativo en las ultimas dos décadas, lo que implica la convivencia con población proveniente de otras ciudades, lo cual trae aparejado el cambio de hábitos y de formas de vida, en particular en la población adolescente. Entre las modificaciones producidas se observa el incremento en el consumo de sustancias adictivas. A pesar de no existir información sobre 
el consumo en adolescentes de la ciudad en años anteriores, existe acuerdo entre las autoridades sanitarias y educacionales y preocupación a nivel general en la sociedad por la relevancia que el problema ha tomado en los últimos tiempos.

En este sentido, la presente investigación contribuye a informar y a proporcionar datos concretos y actualizados sobre el consumo. Se han encontrado cifras significativas en el uso y consumo perjudicial del alcohol, que cobran prominencia si se tienen en cuenta datos como la frecuencia, la cantidad y la pobre conciencia sobre las consecuencias de este consumo excesivo. En relación al consumo de drogas (incluyendo en este criterio drogas legales e ilegales), se observa que las de preferencia son el alcohol y el tabaco, seguidas en menor medida por el consumo de marihuana y medicamentos. La proporción en relación a la elección de las sustancias resulta similar con el consumo de los adolescentes de todo el país, según los datos obtenidos por la Encuesta Nacional a Estudiantes de Enseñanza Media llevada a cabo por la Secretaría de Programación para la Prevención de Drogadicción y Lucha contra el Narcotráfico (2001). Esta encuesta señala que el consumo de alcohol alcanza al $75,1 \%$ de los estudiantes, el de tabaco el $46,7 \%$, psicofármacos sin prescripción médica (estimulantes y tranquilizantes) el 9,5\% y drogas ilegales (principalmente marihuana y otras) el 6,7\%. Como se puede apreciar, los porcentajes de los adolescentes de la ciudad de San Luis que consumen alcohol $(63,6 \%)$ están por debajo de los niveles de consumo nacional, pero señalan una tendencia que se mantiene constante. En cuanto a las diferencias del consumo de alcohol según sexo, los datos obtenidos son similares a los obtenidos por el gobierno nacional, en donde se observa que la tendencia es más marcada en los varones que en las adolescentes mujeres, en el grupo de edades comprendidas entre 15 y 17 años. Un dato que se desprende de la presente investigación y que es coincidente con los resultados de Sedronar (2001), señala que el consumo de sustancias se encuentra legitimado en nuestra sociedad; los jóvenes consideran que está relacionado con situaciones recreativas, como la posibilidad de experimentar y divertirse en situaciones sociales.

Otro de los objetivos propuestos se orientó a identificar estilos de personalidad vinculados al consumo de sustancias. En este sentido, se obtuvo que los adolescentes consumidores tienden a presentar características de personalidad distintivas tal como afirmaban Mesa y León-Fuentes (1996), que influyen en el consumo o pueden predisponer dicha conducta. Estos adolescentes tienden a relacionarse con otros de manera agresiva y displicente, a ser irresponsables e impulsivos, y a presentar un conflicto de ambivalencia entre la obediencia hacia los demás y el negativismo agresivo cuando no obtienen lo que desean. Suelen ser indiferentes a las necesidades de otros y en las relaciones familiares presentan conductas rebeldes y oposicionistas frente a los padres, siendo más frecuente en estos adolescentes la propensión a realizar conductas impulsivas e ilegales. Mesa y León-Fuentes (1996), señalan signos precursores en la infancia o preadolescencia de los sujetos consumidores de sustancias: "...problemas de relación con la familia, fracaso escolar, desarraigo, uso de drogas por otras personas cercanas, conducta delictiva, pobre autoestima, falta de conformidad social y cambios vitales estresantes" (p. 340).

Está muy extendida la hipótesis de que la drogodependencia siempre asienta sobre algún tipo de psicopatología. En este sentido algunos autores coinciden en que los rasgos de personalidad antisocial se vinculan directamente al consumo de drogas. Lacoste (2000), plantea que del total de sujetos que consumen sustancias un porcentaje cercano al $24 \%$ se produce sobre la base de una personalidad antisocial, mientras que el resto surge por la presión de grupo, por las propiedades adictivas de la sustancia y por psicopatología previa de otro tipo. Peluso, Frederick, Foster, Noel y Aglira (1998), refieren que quienes padecen desórdenes de personalidad antisocial requieren una estimulación intensa puesto que tienden a aburrirse o cansarse en las actividades rutinarias y esto los conduce al consumo de alcohol y drogas o a realizar actividades arriesgadas y criminales.

El estudio de Grant, Stinson, Dawson et al. (2004) presenta datos que corroboran que los trastornos por consumo de alcohol y drogas están más relacionados con la personalidad antisocial, histriónica y dependiente.

En conclusión, podemos afirmar que existen rasgos de personalidad vinculados al consumo de sustancias, que influyen y pueden orientar hacia un consumo perjudicial de sustancias adictivas. Estos rasgos de personalidad aportan información fundamental que debe ser profundizada en el trabajo de los profesionales de la salud con adolescentes en ámbitos preventivos, diagnósticos, pedagógicos, clínicos y de investigación; puesto que pueden orientar hacia un mejor conocimiento de la situación del adolescente y de la forma en que se puede efectivizar la ayuda para él.

Resulta importante destacar que los resultados obtenidos para los estudiantes no pueden ser aún generalizados a la población adolescente de nuestra ciudad debido a la limitación en el número de casos. 


\section{REFERENCIAS}

Davis, R. (1999). Millon: Essentials of his science, theory, classification, assessment and therapy. Journal of Personality Assessment, 72, 332, 333.

Florenzano, R. (1992). Crecimiento y desarrollo. En Organización Panamericana de la Salud (OPS) (1992). Manual de medicina del adolescente. Washington: OPS.

Glenn, H. S. (1987). Raising children for success. Fair Oaks, California: Sonrise Press.

Goodman Gilman, A., Hardman, J., Limbird, L., Molinoff, P., Ruddon, R. (1996). Las bases farmacológicas de la terapéutica. México: Mc Graw-Hill Interamericana.

Grant, B. F., Stinson, F. S., Dawson, D. A., Chou, S. P., Ruan, W. J., \& Pickering, R. P. (2004). Cooccurrence of 12month alcohol and drug use disorders and personality disorders in the United States: Results from the National Epidemiologic Survey on Alcohol and Related Conditions. Archives of General Psychiatry, 61, 361368.

Lacoste, J. A. (2000, Marzo). Trastornos mentales asociados al consumo de alcohol y otras drogas. Mesa Redonda presentada en el Primer Congreso Virtual de Psiquiatría.

Mesa, P. y León-Fuentes, J. (1996). Drogodependencias y adolescencia. Causas y consecuencias del abuso. En Buendía, J. (1996). Psicopatología en niños y adolescentes. Madrid: Editorial Pirámide.

Mezzich, A., Tarter, R., Giancola, P., Lu, S., Kirisci, L., y Parks, S. (1997). Substance use and risky sexual behavior in female adolescents. Drug and Alcohol Dependence, 44, pp.157-166.

Millon, T., (1993). Millon Adolescent Clinical Inventory Manual (MACI). Minneapolis, MN: National Computer Systems.

Obiols, G. y Obiols, S. (1994). Adolescencia, Posmodernidad y Escuela Secundaria. Buenos Aires: Editorial Kapelusz.

Organización Mundial de la Salud (1993). Test de Identificación de Desórdenes en el Uso de Alcohol (AUDIT). Ginebra: OMS.

Peláez, P. y Luengo, X. (1996). El adolescente en conflicto. Salud Integral. Santiago de Chile: Editorial Andrés Bello.

Peluso, J., Frederick, S., Foster, A., Noel, A. y Aglira, A. (1998). Desorden de personalidad antisocial. Issues in Psychosocial Nursing, Vol. 1, número 1. Recuperado el 20 de Febrero, http://www.nursing.duq.edu/faculty/ issues/index.html.

Riggs J. (1998). Prevention and Treatment of Alcohol Use Disorders. Washington, DC: American Medical Association: Report of the Executive Council.

Secretaría de Programación para la Prevención de la Drogadicción y la Lucha contra el Narcotráfico (2001). Encuesta Nacional a Estudiantes de Enseñanza Media. Buenos Aires, Argentina: Sedronar.

Selzer, M. L. (1971). The Michigan alcoholism screening test: The quest for a new diagnostic instrument. American Journal of Psychiatry, 127, 1653-1658.

Strack, S. (1999).Special series: Millon's evolving personality theory and measures. Journal of Personality Assessment, 72, 323-325. 